\title{
Editorial
}

\section{Coincidence and Management of Non-Motor Stroke-Associated Risks (Dysphagia - Swallowing Deficits - Visual Neglect)}

In this journal, S.A. Eltringham et al. from the United Kingdom [1] systematically reviewed the methods of assessment and management in patients with acute stroke that influence the risk of stroke-associated pneumonia. Twelve studies of 87,824 patients were included. Protocols related to the types of dysphagia screening and swallowing tests as well as assessment of speech and language protocols with a view to stroke-associated pneumonia varied widely across and within studies. However, it was found that systematic early dysphagia screening plus specialist swallowing assessment reduced stroke-associated pneumonia.

In addition, B. Labeit et al. from Germany [2] reviewed screening parameters for the early detection of post-stroke dysphagia in their own series of 687 patients based on NIHSS and fiberoptic endoscopic dysphagia severity scoring on admission. Both NIHSS and dysphagia severity showed a significant positive correlation in the whole study population $\left(R^{2}=0.745\right)$ as well as in both analysed subgroups $\left(R^{2}=0.494\right.$ for supra- and $R^{2}=0.646$ for in- fratentorial strokes, $p<0.0005$, respectively). For supratentorial strokes, the ideal NIHSS cut-off is $>9$ (sensitivity $68.3 \%$, specificity $61.5 \%$, positive predictive value $89.7 \%$, negative predictive value $28.4 \%$ ). For infratentorial strokes, a lower ideal cut-off $>5$ was calculated (sensitivity $67.4 \%$, specificity $85.0 \%$, positive predictive value $95.1 \%$, negative predictive value $37.8 \%$ ). Thus, NIHSS may be used as an adjunct to predict dysphagia in acute stroke patients with moderate sensitivity and specificity. Differentiation between supra- and infratentorial regions is essential in order not to miss dysphagia in infratentorial stroke.

In this issue, A. Dionísio et al. from Portugal [3] systematically reviewed the applicability of transcranial magnetic stimulation for the recovery of communication skills, swallowing and spatial attention after stroke. The efficacy of transcranial magnetic stimulation as an intervention tool for post-stroke rehabilitation of these nonmotor deficits was confirmed for use in the rehabilitation of aphasia, dysphagia and neglect.

Michael G. Hennerici, Mannheim, Germany

\section{References}

1 Eltringham SA, Kilner K, Gee M, Sage K, Bray B, Pownall S, Smith CJ: Impact of dysphagia assessment and management on risk of stroke-associated pneumonia: a systematic review. Cerebrovasc Dis 2018;46:97105.
2 Labeit B, Mueller H, Muhle P, Claus I, Warnecke T, Dziewas R, Suntrup-Krueger S: Predicting dysphagia with National Institute of Health Stroke Scale: distinction between infra- and supratentorial region is essential. Cerebrovasc Dis 2018;46:150-158.
3 Dionísio A, Duarte IC, Patrício M, CasteloBranco M: Transcranial magnetic stimulation as an intervention tool to recover from language, swallowing and attentional deficits after stroke: a systematic review. Cerebrovasc Dis 2018;46:176-183. 